\title{
Optical and thermal properties of the half-Heusler VFeSb and NbFeSb alloys
}

\author{
K. Bencherif \\ University of AinTemouchent Belhadj Bouchaib, 46000, Algeria. \\ Laboratory of Sudy of Materials and Instrumentations Optics (LEMIO), \\ UDL of Djilali Liabes, SidiBel Abbes, 22000 Algeria. \\ e-mail: bencherifeim@gmail.com
}

Received 31 March 2021; accepted 10 June 2021

In this work, we used the first-principles method FP-LAPW based on density functional theory (DFT) to study the thermal and optical properties of half-Heusler compounds VFeSb and NbFeSb. These materials are characterized with a small and narrow band gap semiconductors close to the Fermi level; following the electron number and other structural properties of these materials, they are expected to have a high thermoelectric performance. In our calculations, we will use the structural and electronic properties for our materials already calculated in previous publications. The modified Becke-Johnson exchange potential (mBJ)-LDA approach was also used. Optical properties such as complex dielectric function, refractive index, reflectivity, and energy loss function for incident photon energy up to $30 \mathrm{eV}$ have been predicted. We also analyze the influence of the pressure and temperature on the primitive cell volume, heat capacity, volume expansion coefficient, and Debye temperature of the Half-Heusler compounds.

Keywords: DFT; (mBJ)-LDA; optical properties; thermal properties; half-Heusler.

DOI: https://doi.org/10.31349/RevMexFis.68.021601

\section{Introduction}

Half-Heusler compounds generally have the composition XYZ [1] and they are ternary alloys with cubic structure where each letter stands for an alloying element, while the complete Heusler are composed according to the pattern $\mathrm{X} 2 \mathrm{YZ}$. By removing one $\mathrm{X}$ atom over two, leaving a vacant site in this context, $\mathrm{X}$ and $\mathrm{Y}$ are transition elements, while $\mathrm{Z}$ is an element of the III-V main group. With intermetallic Half-Heusler phase composed of three elements (X, Y, Z) we can obtain semiconductors materials with small-gap and stable structure when we have 18 electrons in the valence band. The narrow band gap at the Fermi level in some of these compounds and voids in the crystal structure are believed to be the key ingredients making these materials promising candidates for thermoelectric applications, they can be used for the manufacture of several optic sensors and solar cells. The HalfHeusler compounds have attracted particular interest first because of their magnetics and their thermoelectric properties at high temperatures [2-3-4]. In this paper, we study ternary Half-Heusler (SH) compounds Fig. 1 which are composed of transition metals with metalloids $\mathrm{VFeSb}$ and $\mathrm{NbFeSb}$. The optical and thermal properties are calculated by the full potential linearized augmented plane wave (FP-LAPW) method with the modified Becke-Johnson ( $\mathrm{mBJ}$ ) potential. It is also revealed that the effectiveness of $\mathrm{mBJ}$ is based on the proper treatment of the d-orbitals in the highly correlated electron system.

\section{Calculation method}

In our calculations, we use the Wien2k [5] which is a direct application of the FP-LAPW developed by Blaha, Schwarz and their colleagues [6], and which will allow us to study the structural, electronic and optical properties of Half-Heusler compounds XFeSb: $(\mathrm{X}=\mathrm{V}, \mathrm{Nb})$.

The algorithm is based on the density functional theory (DFT) within the local density approximation (LDA) proposed by Perdew and Wang [7] for the exchange correlations functional. It calculates the self-consistent solution of the equations of Kohn and Sham [8] who decry the valence electron in a potential created by a periodic array. The electronic configurations for $\mathrm{V}, \mathrm{Nb}, \mathrm{Fe}$ and $\mathrm{Sb}$ are $\mathrm{V}: \mathrm{Z}$ : 23[Ar] 3d3 4s2, Nb: Z: 41 [Kr] 4d4 5s1, Fe: Z: 26 [Ar] 3d64s2 and $\mathrm{Sb}: \mathrm{Z}: 51[\mathrm{Kr}] 5 \mathrm{~s} 24 \mathrm{~d} 105 \mathrm{p} 3$; In the calculation, the sub shells of the noble gas cores have been distinguished from the sub shells of valence electrons given explicitly.

The $k$ integration over the Brillouin zone is performed using the Monkhorst and Pack mesh [9]. To calculate the band structure of these half-Heusler materials, we used

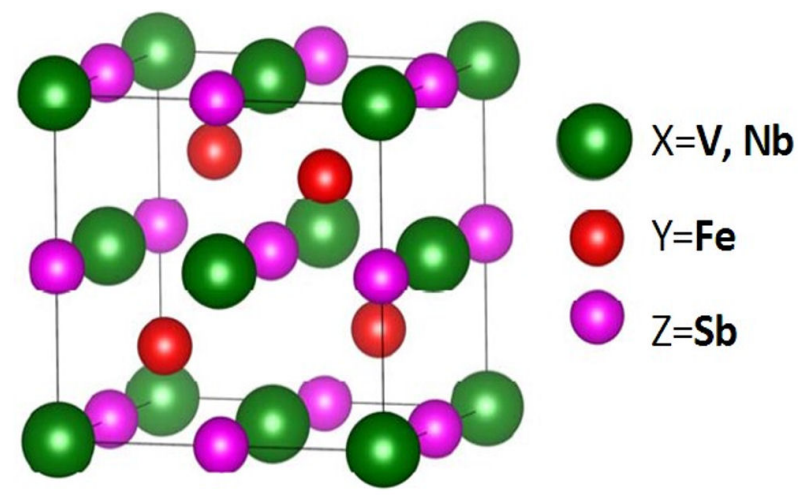

FIGURE 1. The unit cell of the crystal structure of half-Heusler $\mathrm{VFeSb}$ and $\mathrm{NbFeSb}$ compounds. 


$$
v_{x, \sigma}^{m B J}(r)=c v_{x, \sigma}^{B R}(r)+(3 c-2) \frac{1}{\pi} \sqrt{\frac{5}{12}} \sqrt{\frac{2 t_{\sigma}(r)}{\rho_{\sigma}(r)}},
$$

where $v_{x, \sigma}^{m B J}$ is the modified $\mathrm{BJ}$ potential $(\mathrm{mBJ})$,

$$
\rho_{\sigma}=\sum_{i=1}^{N_{\sigma}}\left|\psi_{i, \sigma}\right|^{2}
$$

is the electron density,

$$
t_{\sigma}=\left(\frac{1}{2}\right) \sum_{i=1}^{N_{\sigma}} \nabla \psi_{i, \sigma}^{*} \psi_{i, \sigma},
$$

is the kinetic energy-density,

$$
v_{x, \sigma}^{B R}(r)=-\frac{1}{b_{\sigma}(r)}\left(1-e^{-x_{\sigma}(r)}-\frac{1}{2} x_{\sigma}(r) e^{-x_{\sigma}(r)}\right),
$$

is the Becke-Roussel (BR) potential, and

$$
c=\alpha+\beta\left(\frac{1}{V_{\text {cell }}} \int_{\text {cell }} \frac{\left|\nabla \rho\left(r^{\prime}\right)\right|}{\rho\left(r^{\prime}\right)} d^{3}\left(r^{\prime}\right)\right)^{1 / 2},
$$

was chosen to depend linearly on the square root of the average of $|\nabla \rho| / \rho$ :

The modified BJ exchange potential yields band gaps with accuracy comparable to other approaches, often orders of magnitude more computationally expensive. The potential we propose is given by Eqs. (1)-(5), where $\alpha$ and $\beta$ are two free parameters and $V_{\text {cell }}$ is the unit cell volume. Minimization of the mean absolute relative error for the band gap of the solids listed in Table IIleads to $\alpha=-0.012$ (dimensionless) and $\beta=1.023$ Bohr $^{1 / 2}$.

\section{Results and discussion}

\subsection{Structural properties}

Our half-Heusler are cubic ternary alloys with formula XYZ, where $\mathrm{X}, \mathrm{Y}$ are transition metals, and $\mathrm{Z}$ is a sp-valent element. For the calculation of lattice parameter, we will combine $\mathrm{Y}$ and $\mathrm{Z}$ and $\mathrm{X}$ is changed [11]. VFeSb and $\mathrm{NbFeSb}$, like most of half-Heusler ternary alloys, follow a XYZ structure where atom $\mathrm{X}$ is located at $(0.5,0.5,0.5)$, atom $\mathrm{Y}$ at $(0.25,0.25,0.25)$, and atom $\mathrm{Z}$ at $(0,0,0)$ as can be seen in Fig. 1. To start our calculations we have used the experimental lattice constant. In Table Iwe summarize our calculated lattice parameter $a$ and bulk modulus $B_{0}$ along with its pressure derivative $B^{\prime}$ for $\mathrm{VFeSb}$ and $\mathrm{NbFeSb}$ with the LDA. The lattice constants and bulk modulus are computed by fitting the total energy versus volume according to the Murnaghan's equation of state [12].

When we analyze our results in Table I, we observe that there is good agreement between our results and previous calculations [13].
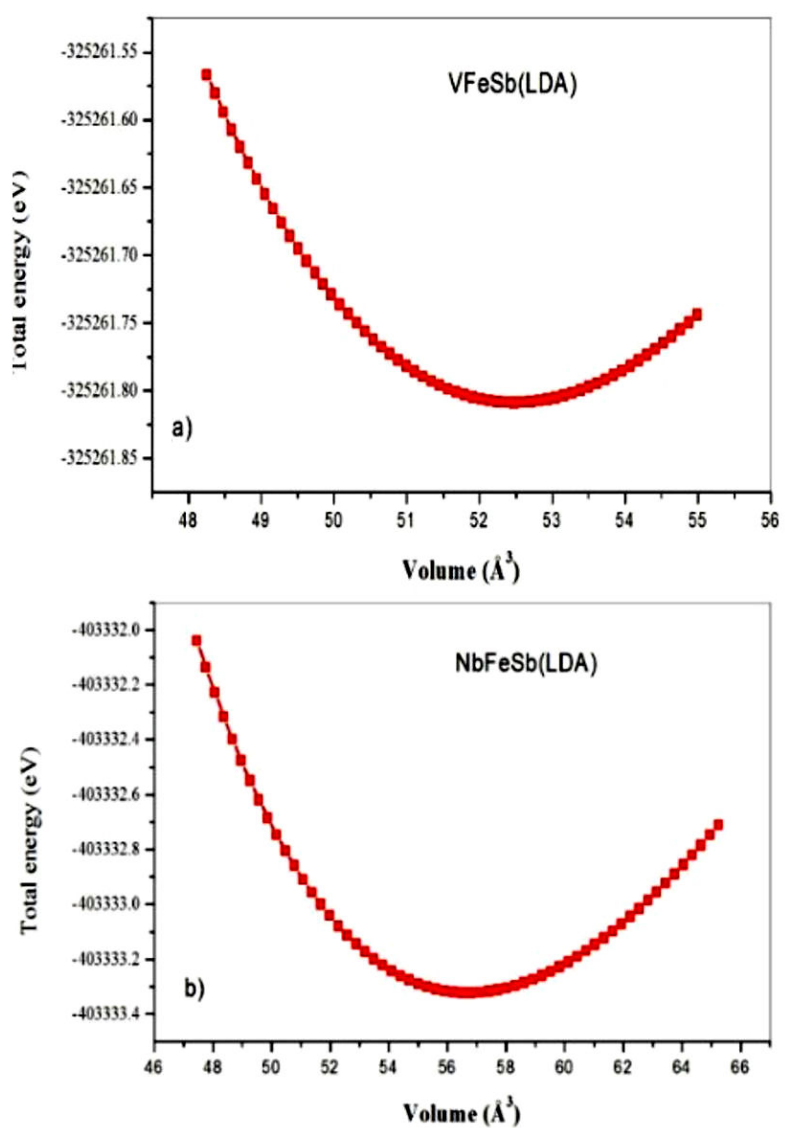

FIGURE 2. Variation of the total energy as a function of the atomic volume of the half-Heusler compound a) $\mathrm{VFeSb}$ and b) $\mathrm{NbFeSb}$ with the LDA.

TABLE I. Calculated lattice parameter $a$, bulk modulus $B_{0}$, and its pressure derivative $B^{\prime}$ for $\mathrm{VFeSb}$ and $\mathrm{NbFeSb}$.

\begin{tabular}{lccc}
\hline Compounds & Lattice $(\AA)$ & $\mathrm{B}_{0}(\mathrm{GPa})$ & $\mathrm{B}^{\prime}$ \\
\hline VFeSb & & & \\
Our calculations & 5.840 & 189.255 & 4.78 \\
Other calculations & $5.957[4]$ & $/$ & \\
NbFeSb & & & \\
Our calculations & 5.901 & 190.163 & 4.59 \\
Other calculations & $5.959[4]$ & $/$ & \\
\hline
\end{tabular}

\subsection{Electronic properties}

\section{Band structure}

In the present work, we study the energy band structure at equilibrium lattice parameters and following that our materials have a very narrow gap, we have used the local density approximation proposed by Perdew and Wang [14] and the modified Becke-Johnson potential [15]. Figure 3 displays the band structure of $\mathrm{VFeSb}$ and $\mathrm{NbFeSb}$, respectively, with LDA approximation and with mBJ. In Table II, we present the gap value obtained from our band structure calculations using 

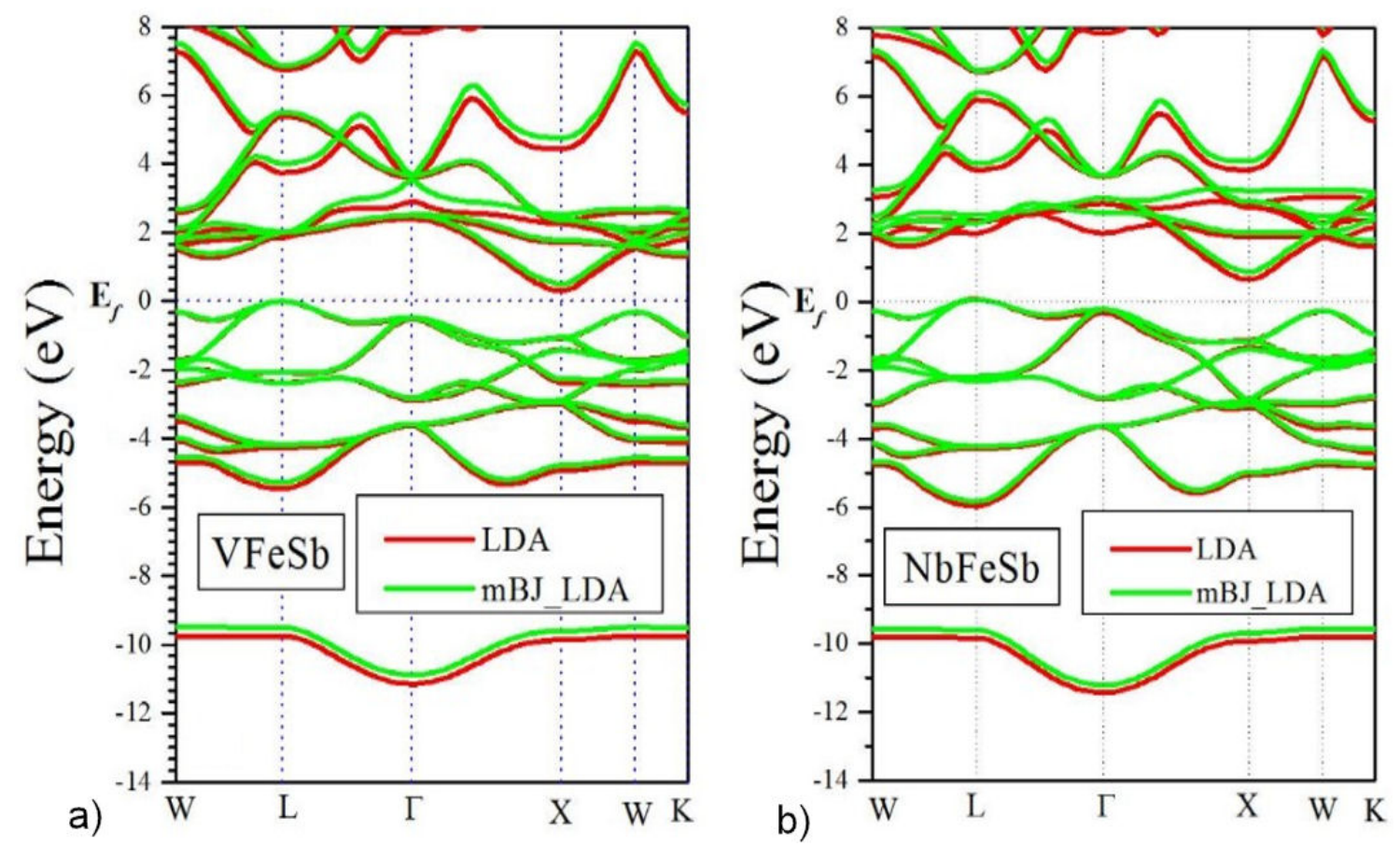

FIGURE 3. Band structure of the a) VFeSb and b) NbFeSb half-Heusler compounds with LDA and MBJLDA.

TABLE II. Band gaps of using LDA and the new MBJLDA of $\mathrm{VFeSb}$ and $\mathrm{NbFeSb}$ (All energies are in $\mathrm{eV}$ ).

\begin{tabular}{lcc}
\hline Compounds & $\mathrm{E}_{g}$ with LDA & $\mathrm{E}_{g}$ with MBJ \\
\hline VFeSb & & \\
Our calculations & 0.302 & 0.583 \\
Other calculations & $0.324[4]$ & $/$ \\
NbFeSb & & \\
Our calculations & 0.543 & 0.991 \\
Other calculations & $0.529[4]$ & $/$ \\
\hline
\end{tabular}

LDA and the new $\mathrm{mBJ}$ along with previous theoretical results. The calculated energy band structure, at equilibrium lattice parameters with the mBJ-LDA, gives us the band gap shown in Fig. 3; $0.583 \mathrm{eV}$ for VFeSb and $0.991 \mathrm{eV}$ for $\mathrm{NbFeSb}$. These values are better than those calculated by the local density approximations. The band gap calculated with the LDA is $0.302 \mathrm{eV}$ for $\mathrm{VFeSb}$ and $0.543 \mathrm{eV}$ for $\mathrm{NbFeSb}$ and is compared in Table II to other calculated values [4].

\section{Density of states (DOS)}

The character of the band states for these materials can be defined by calculating the total and partial state densities DOS using the Tetrahedron method, which was developed by Blöck et al. [16] which requires a large number of special points in the irreducible Brillouin zone.

Figure 4 shows the total densities of states (TDOS) and partial densities of states (PDOS) of the $\mathrm{VFeSb}$ and $\mathrm{NbFeSb}$ compounds calculated by respecting the references of Fermi energy levels which correspond to $0 \mathrm{eV}$. We note the presence of a small gap between the binding states and the anti-binding states in the two VFeSb and $\mathrm{NbFeSb}$. This small gap corresponds to the character of semiconductor compounds. As we can say that between $-5 \mathrm{eV}$ and $0 \mathrm{eV}$ (Fermi level); It's a hybridization of d-states of Vanadium (V) and d-states of Iron $(\mathrm{Fe})$ and $\mathrm{P}$ states of Antimony ( $\mathrm{Sb})$. From this analysis. The intensity of the peaks decreases when the $\mathrm{V}$ is replaced by $\mathrm{Nb}$; Note that all the DOS state densities of the two compounds are similar and come with equal contributions from all the elements X (X: V, Nb) Antimony (Sb) its strong contribution comes from the s orbital between the interval $-12 \mathrm{eV}$ and $-9 \mathrm{eV}$ and with and essentially of the p orbital between $-5 \mathrm{eV}$ and the Fermi level; Above the Fermi level, the density of state is made up of a mixture of s, p orbitals and d. In the vicinity of the Fermi level region, the major contribution to the DOS is due to the d orbital of both Iron $(\mathrm{Fe})$ atoms and $(\mathrm{V}$ and $\mathrm{Nb}$ ). As we can say that between $-5 \mathrm{eV}$ and $0 \mathrm{eV}$ (Fermi level); It's a hybridization of d-states of Vanadium (V) and $\mathrm{d}$-states of Iron $(\mathrm{Fe})$ and $\mathrm{P}$ states of Antimony (Sb). From this analysis, we can suggest that the $d$ states of the electrons of the Iron atoms and $(\mathrm{V}$ and $\mathrm{Nb})$ will play a major role in the transport properties. The phonon dispersion of the compounds: $\mathrm{VFeSb}$ and $\mathrm{NbFeSb}$ have already been studied by other researchers; [3-17-18].

\subsection{Optical properties}

In our calculations, we will use the structural and electronic properties for our materials already calculated previously and summarized in Table I and II [4]. 

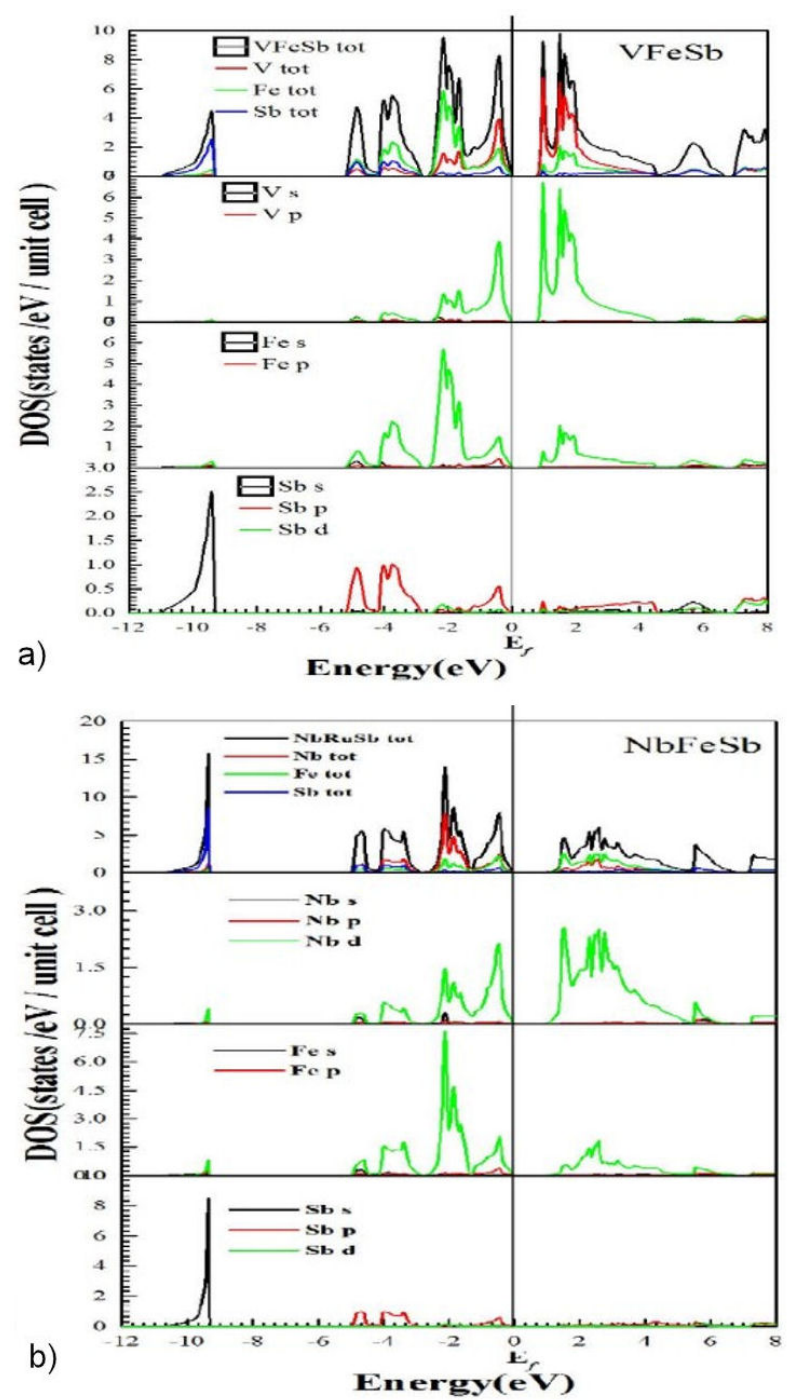

FIGURE 4. The total and partial densities of stat of the VFeSb and $\mathrm{NbFeSb}$ half-Heusler compounds adopting the mBJ-LDA.

The Dielectric function $\varepsilon(\omega)$ has real and imaginary parts,

$$
\varepsilon(\omega)=\varepsilon_{1}(\omega)+i \varepsilon_{2}(\omega) .
$$

The cubic crystal structure of the VFeSb and $\mathrm{NbFeSb}$ half-Heusler compounds only requires the calculation of a single dielectric tensor component to characterize the imaginary part of the dielectric.

For our calculations, we use the exchange-correlation potential MBJ [19].

The imaginary part of the dielectric function $i \varepsilon_{2}(\omega)$ is given by:

$$
\varepsilon_{2}(\omega)=\frac{8}{2 \pi \omega^{2}} \sum_{n \dot{n}} \int_{B Z}\left|P_{n \dot{n}}(k)\right|^{2} \frac{d S_{k}}{\nabla \omega_{n \dot{n}}(k)} .
$$

TABLE III. Optical dielectric constant $\varepsilon(0)$ and refractive index $n(0)$ of $\mathrm{VFeSb}$ and $\mathrm{NbFeSb}$ at zero photon energy.

\begin{tabular}{lcc}
\hline Material & $\varepsilon(0)$ & $n(0)$ \\
\hline $\mathrm{VFeSb}$ & 26.89 & 4.13 \\
$\mathrm{NbFeSb}$ & 26.92 & 4.31 \\
\hline
\end{tabular}

The real part of the dielectric tensor $\varepsilon_{1}(\omega)$ is then determined using the Kramers-Kronig relation

$$
\varepsilon_{1}(\omega)=1+\frac{2}{\pi} P \int_{0}^{\infty} \frac{\dot{\omega} \varepsilon_{2}(\omega)}{\dot{\omega}^{2}-\omega^{2}} d \dot{\omega}
$$

The calculation of important optical functions such as the refractive index $n(\omega)$, the extinction coefficient $k(\omega)$ and energy loss function $L(\omega)$ is performed using the following expressions [20]:

$$
\begin{aligned}
n(\omega) & =\frac{1}{\sqrt{2}}\left(\left[\varepsilon_{1}(\omega)^{2}+\varepsilon_{2}(\omega)^{2}\right]^{1 / 2}+\varepsilon_{1}(\omega)\right)^{1 / 2}, \\
k(\omega) & =\frac{1}{\sqrt{2}}\left(\left[\varepsilon_{1}(\omega)^{2}+\varepsilon_{2}(\omega)^{2}\right]^{1 / 2}-\varepsilon_{1}(\omega)\right)^{1 / 2}, \\
\omega & =\operatorname{Im}\left(-\frac{1}{\varepsilon(\omega)}\right) .
\end{aligned}
$$

The study of optical properties of materials is very important to define the internal structure of the $\mathrm{VFeSb}$ and $\mathrm{NbFeSb}$ compounds. The real and imaginary parts of the dielectric function, $\varepsilon_{1}(\omega)$ and $\varepsilon_{2}(\omega)$,

$$
n(0)=\varepsilon_{1}(\omega)^{1 / 2},
$$

refractive index $n(\omega)$, and loss function $L(\omega)$ are given in Table III and shown in Figs. 5-7 as functions of the photon energy in the range of $0-30 \mathrm{eV}$. The imaginary part of the dielectric function $\varepsilon_{2}(\omega)$ gives the information of absorption behavior of $\mathrm{VFeSb}$ and $\mathrm{NbFeSb}$. The threshold energy of the dielectric function occurs at $E_{0}=0.329 \mathrm{eV}$ for VFeSB and $E_{0}=0.520 \mathrm{eV}$ for $\mathrm{NbFeSb}$, where these energies correspond to the fundamental gap at equilibrium. It is well known that the materials with band small gaps. From Fig. 5, for the imaginary part, it is clear that there are strong absorption peaks in the energy range of $0.329-30 \mathrm{eV}(\mathrm{VFeSb})$ and $0.520-30 \mathrm{eV}$ $(\mathrm{NbFeSb})$. The maximum absorption peak for these materials are, respectively, at $46.66 \mathrm{eV}$ and $44.013 \mathrm{eV}$. The peak around $1.25-25 \mathrm{eV}$ for $\mathrm{VFeSb}$ and $185-25 \mathrm{eV}$ for NbFeSb appear due to the electronic transition from state of the valence band (VB) to the unoccupied state in the conduction band (CB). The real part of the dielectric function $\varepsilon_{1}(\omega)$ is also displayed in Fig. 5. This function $\varepsilon_{1}(\omega)$ gives us information about the electronic polarizability of a material. 


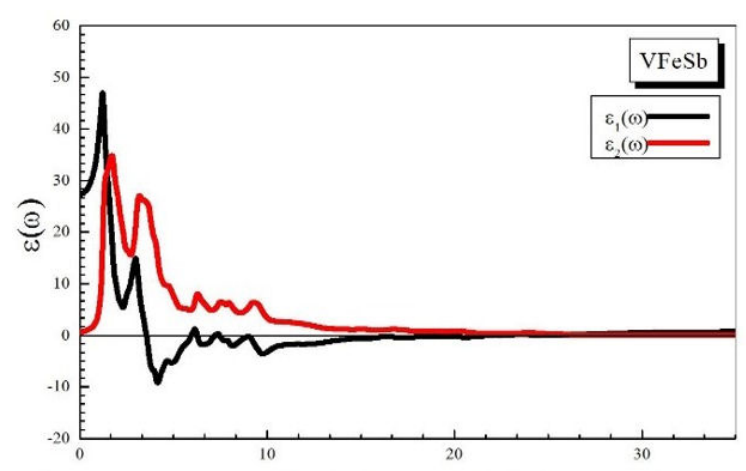

a)

Photon energy (eV)

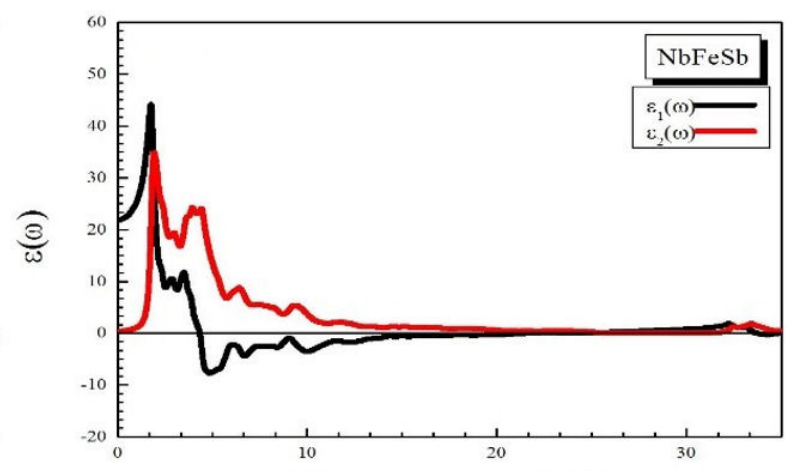

b)

Photon energy (eV)

FIGURE 5. Real and imaginary parts of the dielectric function $\varepsilon(\omega)$ for a) VFeSb and b) NbFeSb with the mBJ-LDA.
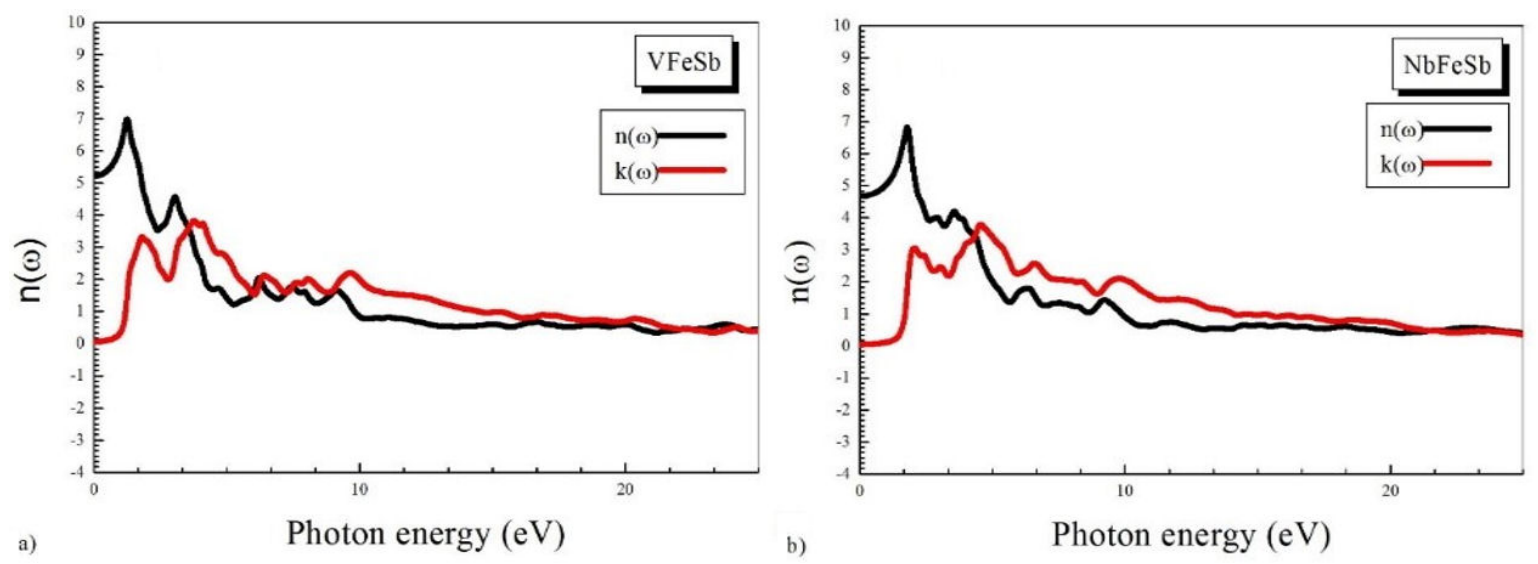

FIGURE 6. Refractive index $n(\omega)$ and extinction coefficient $k(\omega)$ for a) VFeSb and b) NbFeSb with the mBJLDA.

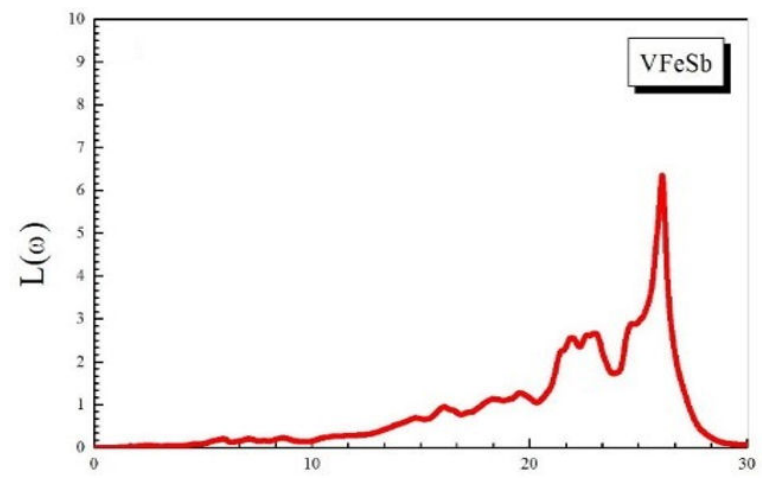

a)

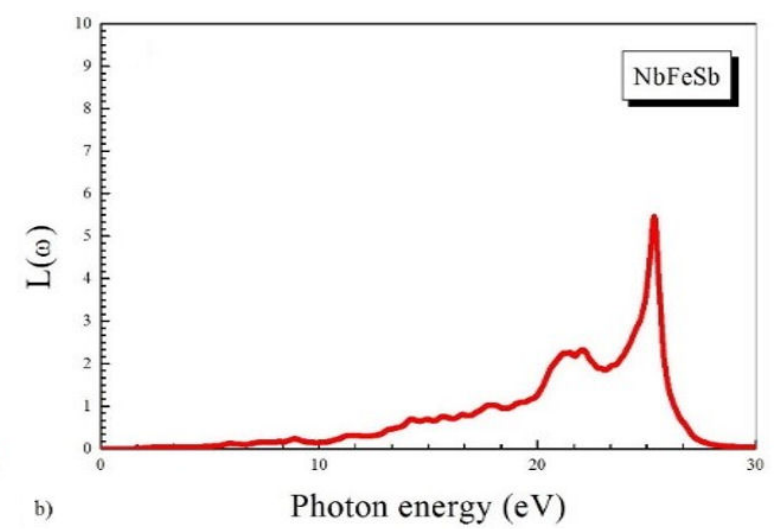

FIGURE 7. Electron energy loss spectra $L(\omega)$ for a) VFeSb and b) NbFeSb with the mBJ-LDA.

The static dielectric constant in its zero frequency limit was found to be $\varepsilon(0)=26.89$ and $\varepsilon(0)=20.92$ for $\mathrm{VFeSb}$ and $\mathrm{NbFeSb}$, respectively. The value of $\varepsilon_{1}(\omega)$ increases as the photon energy increases and reaches a maximum before dropping to a value below the Fermi level, see Fig. 5. The refractive index is displayed in Fig. 6. The static refractive index $n(0)$ is obtained from the real part of the dielectric function, namely,

$$
n(0)=\varepsilon_{1}(\omega)^{1 / 2},
$$

and is found to be 4.1325 and 4.3123 for $\mathrm{VFeSb}$ and $\mathrm{NbFeSb}$, respectively.

The refractive index is larger than 1 as photons penetrate to the material; they are slowed down by the interaction with electrons.

A refractive index of less than unity shows that the group velocity $(V g=c / n)$ of the incident radiation is greater than 
$c$. The energy loss function is displayed in Fig. 7. The energy loss function $L(\omega)$ is an important quantity that describes the energy loss of a fast electron traversing in a material. The peaks in the $L(\omega)$ spectra characterize the plasma resonance. We can see that the main sharp structure of $L(\omega)$ is located at about $15 \mathrm{eV}$, corresponding to a rapid decrease of reflectance. The resonant energy loss is seen at $26.11 \mathrm{eV}$ and $25.37 \mathrm{eV}$ for $\mathrm{VFeSb}$ and for $\mathrm{NbFeSb}$.

\subsubsection{Thermal properties}

We used the quasi-harmonic Debye model (realized in the GIBBS computer code and integrated in the WIEN2k computational package) [21]:

$$
\theta_{D}=\frac{h}{k_{B}}\left(6 \pi^{2} V^{1 / 2} r\right)^{1 / 3} \sqrt{\frac{B_{S}}{M}} \mathcal{F}(\sigma),
$$

where $M$ is the molecular weight per unit volume, $B_{S}$ is the module of the compressibility [22]

$$
B_{S} \cong B(V)=V \frac{d^{2} E(V)}{d V^{2}},
$$

and $f(\sigma)$ is given by the following function [23,24]:

$$
f(\sigma)=\left(3\left[2\left\{\frac{2}{3} \frac{1+\sigma^{3 / 2}}{1-2 \sigma}+\frac{1}{3} \frac{1+\sigma}{1-\sigma}\right\}^{3 / 2}\right]^{-1}\right)^{1 / 3} .
$$

We obtain the pressure and temperature equilibrium by the minimization of the Gibbs function:

$$
\left[\frac{\partial G^{*}(V, P, T)}{\partial V}\right]_{P, T}=0
$$

The heat capacity at constant volume $C_{V}[25]$ is

$$
C_{V}=3 n k\left(4 D\left[\frac{\Theta}{T}\right]-\frac{3 \Theta / T}{e^{\Theta / T}-1}\right) .
$$

The analysis of the thermal properties of materials provides information about their phase stability, melting point, strength, bonding nature, etc. In this work, we calculated the

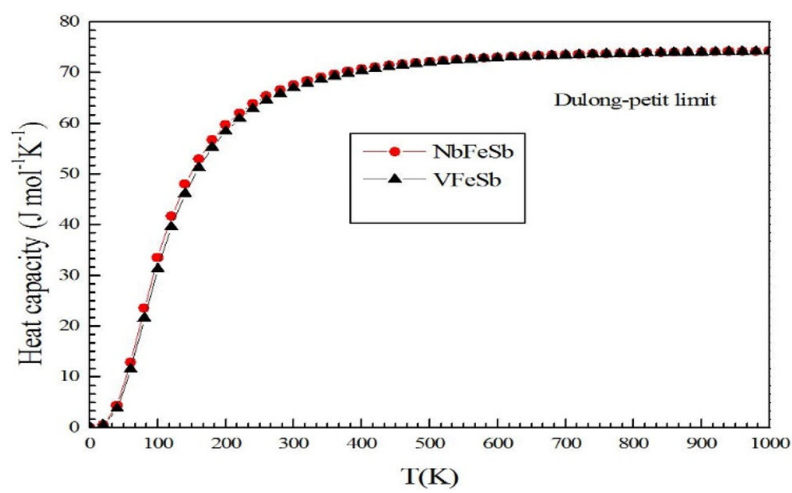

FIGURE 8 . The variation of the heat capacity $C_{V}$ with temperature for the $\mathrm{VFeSb}$ and $\mathrm{NbFeSb}$ compounds.
TABLE IV. The selection of the thermal properties at $300 \mathrm{~K}$ : thermal expansion coefficient (a in $\left.10^{-5} \mathrm{~K}^{-1}\right)$ : $\left(C_{v}\right.$ and $C_{p}$ in $\mathrm{J} / \mathrm{mol}$ $\mathrm{K}$ ), isothermal and adiabatic bulk moduli ( $B$ and $B_{S}$, in GPa), Debye temperature $\left(\theta_{D}\right.$ in $\left.\mathrm{K}\right)$ for $\mathrm{VFeSb}$ and $\mathrm{NbFeSb}$ at zero frequency.

\begin{tabular}{cccccc}
\hline Material & $C_{P}$ & $C_{V}$ & $\mathrm{~B}$ & $\mathrm{~B}_{S}$ & $\theta_{D}$ \\
\hline $\mathrm{VFeSb}$ & 66.03 & 69.04 & 191.45 & 195.34 & 501.22 \\
$\mathrm{NbFeSb}$ & 67.11 & 71.22 & 189.23 & 193.15 & 550.00 \\
\hline
\end{tabular}

thermal properties of the intermetallic half-Heusler, ternary compounds (for $\mathrm{VFeSb}$ and $\mathrm{NbFeSb}$ ) using a density functional theory based approach. To determine the thermal properties, the quasi-harmonic Debye model (realized in the GIBBS computer code and integrated in the WIEN2k computational package) is used. The obtained thermal properties, at room temperature, for the for $\mathrm{VFeSb}$ and $\mathrm{NbFeSb}$ ternary compounds are presented in Fig. 8. It is seen that the for $\mathrm{VFeSb}$ and $\mathrm{NbFeSb}$, ompounds presents the highest heat capacity among the three compounds, as observed in Table IV. On the other hand, the value of the heat capacity $(\mathrm{Cv})$ at higher temperature for all the three crystal structures reaches the value of the classical limit of Dulong and Petit, (69.04 J/molK for $\mathrm{VFeSb}$ and 71.22 J/molK for $\mathrm{NbFeSb}$ ).

\section{Conclusion}

We have presented a theoretical analysis of the different structural, electronic, optical, and thermal properties of $\mathrm{VFeSb}$ and $\mathrm{NbFeSb}$ compounds using the FP-LAPW method with the LDA and mBJ-LDA. The results of the band structure show that the $\mathrm{XFeSb}$ (where $\mathrm{X}=\mathrm{V}, \mathrm{Nb}$ ) compounds with the maximum valence band and the minimum conduction band at the $\mathrm{L}$ and $\mathrm{X}$ symmetry points, respectively, are indirect band-gap semiconductors, which is in good agreement with the previous available studies. The results observed for the density of states may be due to the electro-negativity values (according to the Pauling scale, corresponding to the V, $\mathrm{Nb}, \mathrm{Sb}$ and $\mathrm{Fe}$ atoms, respectively, where they are in close proximity with each other). The outermost electrons of the $\mathrm{Sb}$ atom appear to be polarized to some extent in the direction of the Fe atom. The optical properties such as dielectric function, refractive index, and electron energy loss are studied in the energy range of $0-35 \mathrm{eV}$. The above properties of $\mathrm{VFeSb}$ and $\mathrm{NbFeSb}$ have provided the appearance of several peaks associated with the interband transitions in the two structures. The thermal properties presented in Table IV showcase that our compounds present high Debye temperatures, which indicates that they may present important thermal conductivities. In our calculations the magnetic properties are not considered because our compounds have a small gap, corresponding to a semiconductor behavior, i.e., with vanishing magnetic moments. 
1. J. Pierre et al., Properties on request in Half -Heusler phases, J. Alloys Compd. 262-263 (1997) 101, htts://doi.org/ $10.1016 / \mathrm{S} 0925-8388(97) 00337-\mathrm{X}$

2. H. Hohl et al., Efficient dopants for ZrNiSn-based thermoelectric materials, J. Phys. Condens. Matter 11 (1999) 1697, htts://doi.org/10.1088/0953-8984/11/7/004

3. D. P. Young, P. Khalifah, R. J. Cava, and A. P. Ramirez, Thermoelectric properties of pure and doped FeMSb $(\mathrm{M}=\mathrm{V}, \mathrm{Nb}) ; J$. Appl. Phys. 87 (2000) 317. htts://doi.org/10.1063/ 1.371863

4. J. Yang, Evaluation of Half-Heusler Compounds as Thermoelectric Materials Based on the Calculated Electrical Transport Properties, Adv. Funct. Mater. 18 (2008) 2880. htts : //doi.org/10.1002/adfm.200701369

5. P. Blaha, K. Schwarz, G. Madsen, D. Kvasnicka, and J. Luitz, WIEN2k: An Augmented Plane Wave+Local Orbitals Program for Calculating Crystal Properties, htts://euler.phys. cmu.edu/cluster/WIEN2k/usersguide.pdf

6. M. Hachemaoui et al., FP-APW + lo study of the elastic, electronic and optical properties of the filled skutterudites $\mathrm{CeFe}_{4} \mathrm{As}_{12}$ and $\mathrm{CeFe}_{4} \mathrm{Sb}_{12}$, Curr. Opin. Solid State Mater. Sci. 13 (2009) 105. htts://doi.org/10.1016/ j.cossms.2009.04.004

7. J. P. Perdew and Y. Wang, Accurate and simple analytic representation of the electron-gas correlation energy; Phys. Rev. B $\mathbf{4 5}$ (1992) 13244. htts://doi.org/10.1103/PhysRevB. 45.13244

8. W. Kohn and L. J. Sham, Self-Consistent Equations Including Exchange and Correlation Effects Phys. Rev. 140 (1965) A1133. htts://doi.org/10.1103/PhysRev. 140.A1133

9. H. J. Monkhorst and J. D. Pack, Special points for Brillouinzone integrations; Phys. Rev. B 13 (1976) 5188, htts:// doi.org/10.1103/PhysRevB.13.5188

10. F. Tran and P. Blaha, Accurate Band Gaps of Semiconductors and Insulators with a Semilocal Exchange-Correlation Potential. Phys. Rev. Lett. 102 (2009) 226401. htts : / / doi .org/ 10.1103/PhysRevLett.102.226401

11. W. Feng, D. Xiao, Y. Zhang, and Y. Yao, Half-Heusler Topological Insulators: A First-Principle Study with the TranBlaha Modified Becke-Johnson Density Functional; Phys. Rev. B 82 (2010) 235121, htts://doi.org/10.1103/ PhysRevB.82.235121

12. F. D. Murnaghan, The Compressibility of Media under Extreme Pressures, Proc. Natl. Acad. Sci. USA 30 (1944) 5390. https://doi.org/10.1073/pnas.30.9.244

13. P. Villars, L. D. Calvert and W. B. Pearson, Pearson's handbook of crystallographic data for intermetallic phases. (American Society for Metals. Metals Park. 1985).
14. J. P. Perdew and Y. Wang, Phys. Rev. B 45 (1992) 13244. htts://doi.org/10.1103/PhysRevB.45.13244

15. W. Kohn and L. J. Sham, Self-Consistent Equations Including Exchange and Correlations Effects, Phys. Rev. 140 (1965) A1133. htts://doi.org/10.1557/PROC-278-313

16. P. E. Blöchl, O. Jepsen, and O. K. Anderson, Improved tetrahedron method for Brillouin-zone integration, Phys. Rev. B 49 (1994) 16223. htts://doi.org/10.1103/PhysRevB. 49.16223

17. C. Çoban, K. Çolakoglu and Y. Ö. Çiftçi, First principles study of the structural, mechanical, phonon, optical, and thermodynamic properties of half- Heusler $(\mathrm{HH})$ compound NbFeSb, Phys. Scr. 90 (2015) 095701, https : / /doi .org/ 10.1088/0031-8949/90/9/095701

18. A. Page, P.F.P. Poudeu, and C. Uhera, A first-principles approach to half- Heusler thermoelectrics: Accelerated prediction and understanding of material properties, J. Materiomics, 2 (2016) 104. htts://doi.org/10.1016/j. jmat.2016.04.006

19. J. Sun, H. Wang, J. He, Y. Tian, Ab initio investigations of optical properties of the high-pressure phases of $\mathrm{ZnO}$, Phys. Rev. B 71 (2005) 125132. htts://doi.org/10.1103/ PhysRevB.71.125132

20. Y. Huan, C. Jing, Li. Zhe, and X.-R. Chen, First-principles calculations for electronic and optical properties of the zinc blende structured BeS compound under pressure; Chin. Phys. B 18 (2009) 10. htts://iopscience.iop.org/article/ $10.1088 / 1674-1056 / 18 / 10 / 056 / \mathrm{pdf}$

21. X.-W. Sun et al., Heat Capacity and Gruneisen Parameter for GaN with Zinc-Blende Structure; Chin. J. Chem. Phys. 20 (2007) 233. htts://doi.org/10.1088/1674-0068/ 20/03/233-236

22. Z. L. Lv, Y. Cheng, X. R. Chen, and G. F. Ji, Electronic, elastic and thermal properties of $\mathrm{SrCu}_{2} \mathrm{As}_{2}$ via first principles calculation, J. Alloys Compd. 570 (2013) 156, htts: //doi.org/ $10.1016 / j \cdot j a l l c o m .2013 .03 .132$

23. M. A. Blanco, E. Francisco, and V. Luaña, GIBBS: isothermalisobaric thermodynamics of solids from energy curves using a quasi-harmonic Debye model, Compt. Phys. Commun., 158 (2004) 57, htts://doi.org/10.1016/j. comphy.2003.12.001

24. F. Q. Wang, Y. Guo, Q. Wang, Y. Kawazoe, and P. Jena, Exceptional Thermoelectric Properties of Layered GeAss 2 , Chem. Mater. 29 (2017) 9300. htts://pubs.acs.org/doi/ 10.1021/acs.chemmater.7b03279

25. B. Boucher et al., Enhancement of the Thermoelectric Properties of $\mathrm{FeGa}$-type Structures with Group 6 Transition Metals: A Computational Exploration, Inorg. Chem. 56 (2017) 4229. htts : / / pubs . acs.org/doi/abs/10. 1021/acs.inorgchem.7b00251 